\title{
Sorafenib-Related Adverse Events in Predicting the Early Radiologic Responses of Hepatocellular Carcinoma
}

\author{
Shou-Wu Lee ${ }^{a}$, b, d, Teng-Yu Lee ${ }^{a}$, b, Sheng-Shun Yang ${ }^{a}$, c, Chun-Fang Tong ${ }^{a}$, \\ Hong-Zen Yeh" ${ }^{\text {a c }}$, Chi-Sen Chang ${ }^{\text {a, b }}$
}

\begin{abstract}
Background: Hepatocellular carcinoma (HCC) has a poor prognosis with low chemotherapeutic efficiency to medications except to sorafenib. Previous studies showed that adverse events (AEs) of sorafenib can predict therapy efficacy to HCC. The aim of the study is to evaluate the early efficacy and AEs of sorafenib therapy.

Methods: The database of HCC patients receiving sorafenib at Taichung Veterans General Hospital during the period from June 2012 to October 2016 was analyzed. All HCC cases were Barcelona Clinic Liver Cancer (BCLC) classification stage C. The early efficacy of sorafenib was classified according to the mRECIST criteria as either partial response (PR), stable disease (SD) or progressive disease (PD). Responses were recorded within 6 weeks after the start of sorafenib treatment. AEs were defined as the appearance of hand-foot skin reaction (HFSR), hypertension (HTN) and diarrhea. Exclusion criteria were poor performance status, poor drug compliance, discontinued follow-up or mortality occurring within 1 day after medication.
\end{abstract}

Results: From a total of 222 subjects, eight cases (3.6\%) were classified as PR, 82 cases (36.9\%) SD, and 132 cases (59.5\%) PD. The PR group had the highest ratio of HFSR (62.4\%) and hypertension (37.5\%). Pooling cases of PR and SD together, the presence of HFSR adjusted odd ratio (aOR) 2.80, 95\% confidence interval (CI) 1.52 5.16 ) and diarrhea (aOR $3.42,95 \%$ CI 1.67 - 7.01) were good predictors of favorable responses to sorafenib therapy.

Conclusions: HFSR and diarrhea are good predictors of early therapy efficacy to the sorafenib treatment.

Keywords: Hand-foot skin reaction; Hepatocellular carcinoma; Sorafenib

Manuscript submitted November 2, 2018, accepted December 10, 2018

aDivision of Gastroenterology, Department of Internal Medicine, Taichung Veterans General Hospital, Taichung, Taiwan

bepartment of Internal Medicine, Chung Shan Medical University, Taichung, Taiwan

'Department of Internal Medicine, Yang-Ming University, Taipei, Taiwan

${ }^{\mathrm{d} C}$ Corresponding Author: Shou-Wu Lee, Division of Gastroenterology, Department of Internal Medicine, Taichung Veterans General Hospital, Taichung, 1650 Taiwan Boulevard, Sec. 4, Taichung 40705, Taiwan.

Email: ericest@vghtc.gov.tw

doi: https://doi.org/10.14740/gr1109

\section{Introduction}

Hepatocellular carcinoma (HCC) is the commonest primary liver cancer. The Barcelona Clinic Liver Cancer staging system (BCLC) is widely used for selecting its treatment, which is determined collectively by the tumor characteristics, such as size, number, presence of vascular invasion or extrahepatic metastasis, and the hepatic function and performance status of the patient [1]. Advanced HCC, such as BCLC stage C, is typically treated with sorafenib, which is an orally administered inhibitor of multiple protein kinases (such as c-Raf, B-Raf, mitogen-activated protein kinase kinase, extracellular signal regulated kinase, and vascular endothelial growth factor) [2]. The studies of phase III SHARP trial and Asia-Pacific trial regarding sorafenib treatment of advanced HCC patients both reported improvements compared with placebo in terms of their median overall survival (OS) and time to progression (TTP) $[3,4]$.

Although sorafenib is currently the recommended first-line medication for patients with BCLC stage C HCC, a substantial number of patients (with a disease-control rate as as high as $43 \%$ ) fail to respond to the sorafenib [3]. Good predictive factors for the sorafenib efficacy remain unclear. Analyses of serological markers in patients participating in the SHARP trial, showed that the serum concentrations of vascular endothelial growth factor (VEGF) and angiopoietin-2 are good predictors of the patient survival, although not good predictors on the response to treatment [5]. On the contrary, some studies on HCC patients reported the adverse events (AEs) of sorafenib treatment, such as diarrhea, hand-foot syndrome reaction (HFSR) and hypertension (HTN), may predict the efficacy of medication [6-8].

The aim of the present study is to determine what factors can influence the efficacy of sorafenib in terms of the occurrence of common AEs like HFS, hypertension and diarrhea.

\section{Materials and Methods}

Data for the subjects with $\mathrm{HCC}$ receiving sorafenib at Taichung Veterans General Hospital from June 2012 to October 2016 were evaluated. HCC was diagnosed according to the American Association for the Study of Liver Disease (AASLD) guideline [9]. All cases were stage $\mathrm{C}$ HCC determined with the BCLC classification. Data of the enrolled patients included the 
Table 1. The General Data of Each Group With Different Radiologic Responses With Sorafenib

\begin{tabular}{|c|c|c|c|c|c|c|c|c|c|c|}
\hline & \multicolumn{3}{|c|}{ PR group $(N=8)$} & \multicolumn{3}{|c|}{ SD group $(N=82)$} & \multicolumn{3}{|c|}{ PD stage $(\mathrm{N}=132)$} & \multirow{2}{*}{ P-value } \\
\hline & $\mathbf{M} \pm \mathbf{S D}$ & $\mathbf{N}$ & $\%$ & $\mathbf{M} \pm \mathbf{S D}$ & $\mathbf{N}$ & $\%$ & $\mathbf{M} \pm \mathbf{S D}$ & $\mathbf{N}$ & $\%$ & \\
\hline Age (years) & $67 \pm 10.36$ & & & $65.27 \pm 13.44$ & & & $63.8 \pm 11.53$ & & & $0.355^{\mathrm{a}}$ \\
\hline BCLC stage C & & 8 & $100 \%$ & & 82 & $100 \%$ & & 132 & $100 \%$ & $1.000^{\mathrm{b}}$ \\
\hline PVT & & 2 & $25.00 \%$ & & 45 & $54.90 \%$ & & 79 & $59.80 \%$ & $0.141^{\mathrm{b}}$ \\
\hline $\mathrm{HCV}$ & & 6 & $75.00 \%$ & & 36 & $43.90 \%$ & & 49 & $37.10 \%$ & $0.085^{\mathrm{b}}$ \\
\hline Bilirubin (U/L) & $0.7 \pm 0.35$ & & & $0.83 \pm 0.39$ & & & $0.92 \pm 0.47$ & & & $0.084^{\mathrm{a}}$ \\
\hline $\operatorname{ALT}(\mathrm{U} / \mathrm{L})$ & $84.25 \pm 71.39$ & & & $69.03 \pm 62.45$ & & & $60.58 \pm 54.16$ & & & $0.220^{\mathrm{a}}$ \\
\hline $\operatorname{AFP}\left(\times 10^{4} \mathrm{ng} / \mathrm{mL}\right)$ & $3.11 \pm 6.31$ & & & $7.76 \pm 26.45$ & & & $24.52 \pm 69.45$ & & & $0.012^{\mathrm{a}}$ \\
\hline
\end{tabular}

aP-values were analyzed with independent $t$-test; ${ }^{b}$ Pearson's Chi-square test. AFP: alpha-fetoprotein; ALT: alanine aminotransferase; BCLC: BarceIona Clinic Liver Cancer staging system; HBV: hepatitis B; HCV: hepatitis C; HFSR: hand-foot syndrome reaction; HTN: hypertension; M: mean; N: number of patients; PD: progressive disease; PR: partial response; PVT: portal vein thrombosis; SD: stable disease or standard derivation.

following items: age, gender, presence of chronic hepatitis B (HBV) or hepatitis $\mathrm{C}(\mathrm{HCV})$ infection, $\mathrm{HCC}$ with portal vein thrombosis (PVT) or extra-hepatic metastasis, serum level of bilirubin, alanine aminotransferase (ALT) and alpha-fetoprotein (AFP). The initial dosage of sorafenib of each patient was also recorded. Exclusion criteria were cirrhosis HCC BCLC stage A, B or D, decompensated liver status, poor performance status, lack of compliance to drug, loss of follow-up or mortality within 1 day after medication.

According to the mRECIST criteria [10], the early radiologic efficacy of sorafenib was classified as complete response (CR), partial response (PR), stable disease (SD) and progressive disease (PD), as observed within a period of 6 weeks after the beginning of medication. CR was defined as disappearance of all HCC lesions; PR is defined as at least a $30 \%$ decrease in the sum of the diameters of variable HCC lesions; SD is defined as any cases that do not qualify for either PR or PD; PD is defined as an increase of at least $20 \%$ in the sum of the diameters of variable HCC lesions. AEs included the appearance of HFSR, HTN, or diarrhea. The AE of HTN is defined as new-onset of HTN (>140/90 mm Hg) in the cases without underline HTN, or more increased blood pressure that need addition medications in those with underline HTN. The associations between AEs and the efficacy of sorafenib were analyzed.

Data were expressed as standard deviation of mean for each of the measured parameters. Gender and positive ratio of each stratified group were expressed as the percentage of total patient number. Statistical comparisons were made using Pearson's Chi-square test to determine the effects of gender and positive ratio of each stratified group. Independent $t$ test was used to analyze age, serum bilirubin, ALT, AFP and daily sorafenib dosage. Statistical significance was set at $\mathrm{P}$ $<0.05$. Multivariate Cox's regression was used to determine the strength of association between the individuals with each sorafenib-associated AE and the sorafenib efficacy, as shown by odd ratios (OR) with 95\% confidence interval (CI).

\section{Results}

From a total of 222 enrolled subjects, eight $(3.6 \%)$ of them belonging to group PR, 82 (36.9\%) belonging to group SD, and $132(59.5 \%)$ belonging to group PD. No case was classified as radiologic CR. The general data of each group are listed in Table 1. Their average ages ( 67.00 vs. 65.27 vs. 63.80 years, $\mathrm{P}=0.355)$ were similar, so were their gender distributions (male ratios: $87.5 \%$ vs. $82.9 \%$ vs. $86.4 \%, \mathrm{P}=0.775$ ). The ratio of PVT was as follows, group PR: $25.0 \%$, group SD: $54.9 \%$, and group PD: $59.8 \%$. Their extra-hepatic metastasis was group PR: $75.0 \%$, group SD: $53.7 \%$, and group PD: $51.5 \%$. The occurrence of HBV was group PR: $37.5 \%$, group SD: $45.1 \%$, and group PD: $50.8 \%$. Their HCV was group PR: $75.0 \%$, group SD: $43.9 \%$, and group PD: $37.1 \%$. These intergroup differences were not statistically significant. The levels of serum bilirunin and ALT were similar across groups. The PD group showed the significant highest average serum level of AFP $\left(24.52 \times 10^{4} \mathrm{ng} / \mathrm{mL}\right.$ in PD group vs. $3.11 \times 10^{4} \mathrm{ng} / \mathrm{mL}$ in PR group vs. $7.76 \times 10^{4} \mathrm{ng} / \mathrm{mL}$ in SD group, $\mathrm{P}=0.012$ ). The average daily dosage of sorafenib was $3.50 \times 200 \mathrm{mg}$ in group $\mathrm{PR}, 3.48 \times 200 \mathrm{mg}$ in group SD, and $3.29 \times 200 \mathrm{mg}$ in group $P D$, with no significant differences across groups $(P=0.745)$.

AEs detected in each group are shown in Table 2. Among all subjects, 78 cases $(36.9 \%)$ had HFRS, 23 cases $(10.4 \%)$ had HTN and 51 cases $(23.0 \%)$ had diarrhea. The PR group had the highest and significant ratio of HFSR $(62.4 \%$ in PR group vs. $48.8 \%$ in SD group vs. $25.0 \%$ in $\mathrm{PD}$ group, $\mathrm{P}=0.001$ ) and HTN $(37.5 \%$ in PR group vs. $13.4 \%$ in SD group vs. $6.9 \%$ in $\mathrm{PD}$ group, $\mathrm{P}=0.025)$, compared with other groups. On the contrary, the SD group had the highest and significant ratio of diarrhea (34.6\% in SD group vs. $25.0 \%$ in PR group vs. $15.9 \%$ in $\mathrm{PD}$ group, $\mathrm{P}=0.004$ ). 
Table 2. The Ratio of Adverse Events Detected in Each Group

\begin{tabular}{|c|c|c|c|c|c|c|c|c|c|c|}
\hline & \multicolumn{2}{|c|}{ PR group $(N=8)$} & \multicolumn{2}{|c|}{ SD group $(N=82)$} & \multicolumn{2}{|c|}{ PD stage $(\mathrm{N}=132)$} & \multirow{2}{*}{ P-value ${ }^{a}$} & \multirow{2}{*}{ P-value ${ }^{b}$} & \multicolumn{2}{|c|}{ Overall $(\mathrm{N}=222)$} \\
\hline & $\mathbf{N}$ & $\%$ & $\mathbf{N}$ & $\%$ & $\mathbf{N}$ & $\%$ & & & $\mathbf{N}$ & $\%$ \\
\hline HFSR & 5 & $62.40 \%$ & 40 & $48.80 \%$ & 33 & $25.00 \%$ & 0.001 & 0.001 & 33 & $36.90 \%$ \\
\hline Diarrhea & 2 & $25.00 \%$ & 28 & $34.60 \%$ & 21 & $15.90 \%$ & 0.007 & 0.002 & 21 & $23.00 \%$ \\
\hline
\end{tabular}

All P-values were analyzed with Pearson's Chi-square test; aP-value between PR group and PD group; bP-valued between SD group and PD group. HFSR: hand-foot syndrome reaction; HTN: hypertension; N: number of patients; PD: progressive disease; PR: partial response; SD: stable disease.

The statistical associations between AEs and therapeutic responses are listed in Table 3. After adjustments for age, sex and serum AFP, the incidences of HFSR (adjusted OR 5.76, 95\% CI 1.19 - 27.88) and HTN (adjusted OR 7.68, 95\% CI 1.50 - 39.23) showed a positive and significant correlation with sorafenib therapy response in group PR. AEs of HFSR (adjusted OR 2.60, 95\% CI 1.39 - 4.87) and diarrhea (adjusted OR 3.54, 95\% CI 1.70 - 7.40) had significant associations with sorafenib therapy response in group SD. Combining cases in groups PR and SD, the incidences of HFSR (adjusted OR 2.80, 95\% CI 1.52 - 5.16) and diarrhea (adjusted OR 3.42, 95\% CI 1.67 - 7.01) were found to be predictive of good sorafenib therapy response.

\section{Discussion}

Sorafenib is an orally active multikinase inhibitor that is known to prolong OS and TTP in patients with advanced HCC $[3,4]$. Common sorafenib-related AEs are diarrhea, fatigue, anorexia, HTN and dermatological toxicities, mainly HFSR. In the SHARP trial, the overall incidence of treatment-related
AEs is $80 \%$ in the sorafenib group (versus $52 \%$ in the placebo group), with serious AEs of $52 \%$ in the treated group (versus $54 \%$ in the placebo group). The grade 3 treatment-related AEs are more frequent in the sorafenib group: including diarrhea $(8 \%)$, HFSR (8\%) and HTN (2\%) [3]. In the Asia-Pacific tri$\mathrm{al}$, the overall incidence of treatment-related AEs is $81.9 \%$ in the sorafenib group (versus $38.7 \%$ in the placebo group), and the most frequent grade 3/4 drug-related AEs in the sorafenib group are HFSR $(10.7 \%)$, diarrhea $(6.0 \%)$ and fatigue $(3.4 \%)$ [4].

The percentages of AEs in our sorafenib-treated groups were: $36.9 \%$ HFSR, $10.4 \%$ HTN and $23.0 \%$ diarrhea. These proportions are higher than those reported in the above mentioned clinical trials. The discrepancy in results might be due to that in our study we adopted the self-reported design and analyzed all grade AEs.

HFSR is characterized by erythema, dysesthesia or paresthesia on the palms and soles, together with rash. This host of symptoms suggested the involvement of inhibition in one or more of these receptors/pathways (such as VEGFR, platelet derived growth factor receptor (PDGFR), c-KIT or FLT-3) in the development of HFSR [11]. Since sorafenib leads to tu-

Table 3. Odd Ratios and 95\% Confidence Interval of Associated Adverse Events With Sorafenib Response

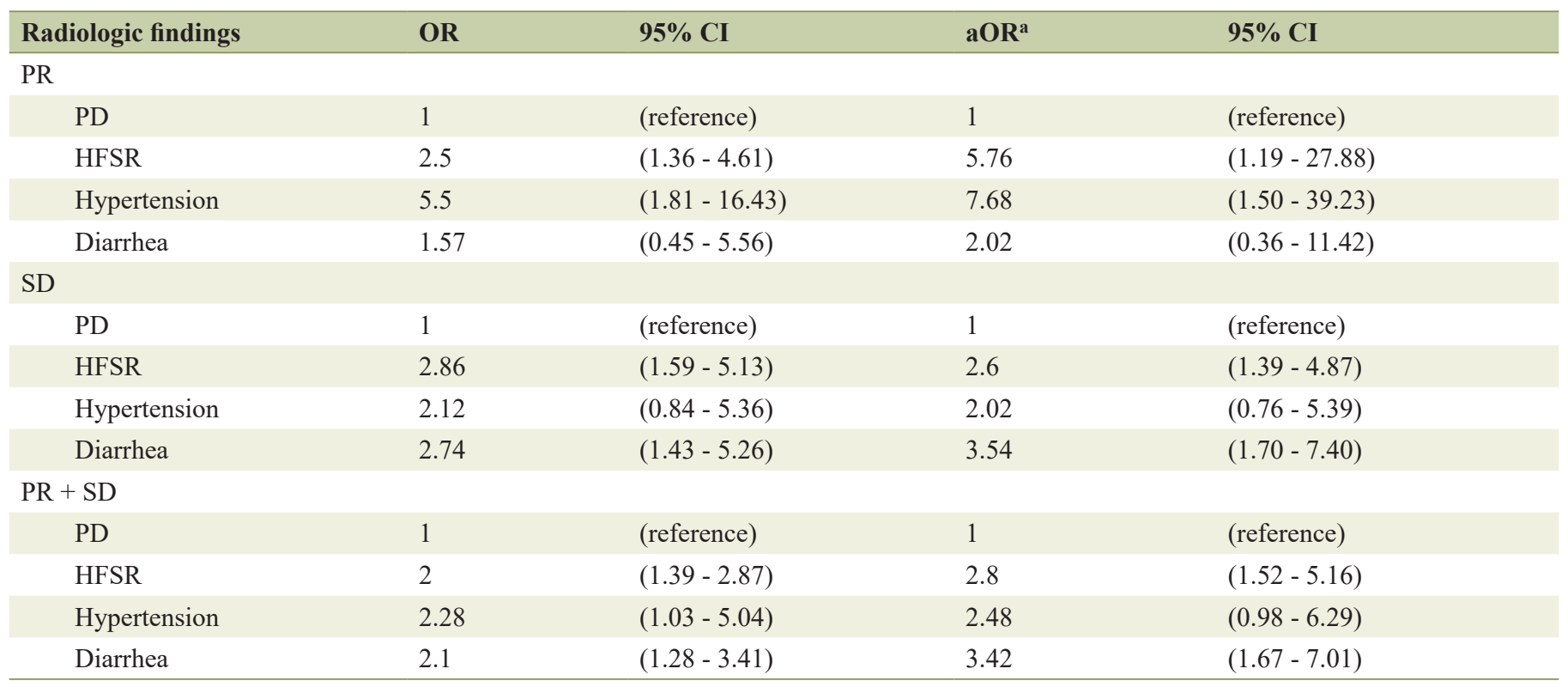

aaOR: adjusted OR (adjusted for age, sex, AFP and sorafenib dosage). Analyzed with Multivariate Cox's regression. Cl: confidence interval; HFSR: hand-foot syndrome reaction; HTN: hypertension; OR: odd ratio; PD: progressive disease; PR: partial response; SD: stable disease. 
mor vessel regression which could inhibit endothelial cells, the capillary endothelium might be the first target in HFSR development [6]. In a previous study in Japan, patients who had developed HFSR (62\%) survive significantly longer than those without cutaneous AEs (OR 0.449, 95\% CI 0.256 - 0.786, P $=0.005)$ [12]. In a Korean retrospective study of 99 patients with advanced HCC (BCLC stage $\mathrm{C}$ ), the presence of HFSR is predictive of a longer TTP $(\mathrm{OR} 0.40,95 \%$ CI $0.19-0.82, \mathrm{P}=$ 0.007 ) and better OS (OR 0.40; 95\% CI $0.24-0.67 ; \mathrm{P}=0.001)$ [7]. These above findings are however in contradiction to other studies that reported that HFSR has no prognostic significance $[8,13]$.

For our patients, the adjusted OR for PR was $5.76(95 \%$ CI 1.19 - 27.88), and for SD was 2.60 (95\% CI 1.39 - 4.87). Those with HFSR had therefore better sorafenib efficacy compared with those without, when judged based on a short-term radiologic presentation.

Arterial HTN is considered a class-specific toxicity of antiangiogenic treatments. Impaired angiogenesis could result in fewer microvessels, and endothelial dysfunctions due to reduced nitric oxide production and the activation of the endothelin-1 system, which is a potent vasoconstrictor [6]. One study enrolling 41 patients with advanced $\mathrm{HCC}$ who received sorafenib, and the result found significantly longer OS in patients with HTN regardless of its grade than in patients without HTN during treatment (median OS 18.2 vs. 4.5 months, $\mathrm{P}=$ $0.016)$ [14]. Another study enrolling 38 patients with advanced HCC disclosed HTN, that occurred within the first 2 weeks following the start of sorafenib treatment, is correlated with a better TTP (153 vs. 50.5 days, $\mathrm{P}=0.017)$ and OS (1,329 vs. 302 days, $\mathrm{P}=0.003$ ) [15]. However, another study concluded on the contrary that treatment-related HTN shows no correlation with clinical outcomes [12].

Our results showed that the presentation of HTN was correlated with the responses in PR group (adjusted OS 7.68, 95\% CI 1.50 - 39.23), but not in the SD group (adjusted OS 2.02, $95 \%$ CI 0.76 - 5.39). When cases from the PR and SD groups were combined for analysis, HTN showed no prognostic significance (adjusted OS 2.48, 95\% CI 0.98 - 6.29).

As VEGF plays a role in maintaining the normal adult vasculature, inhibiting VEGFR with sorafenib could cause diarrhoea via reduction of capillary networks in the intestinal villi [6]. Other hypotheses speculate that sorafenib cause diarrhoea by inducing pancreatic exocrine dysfunction, since VEGFR inhibitors reduce the density of the capillaries in pancreatic islets and decrease zymogen granules [6]. One retrospective study enrolling 112 patients with advanced HCC reported through multivariate Cox regression analysis, that diarrhea is an independent positive prognostic factor $(\mathrm{OR} 0.41, \mathrm{P}=0.001)$ and those cases with diarrhea have a significantly longer median OS than those without (14.1 vs. 7.1 months, $\mathrm{P}=0.011)$ [8]. Another sorafenib prospective study on 46 patients with advanced HCC reported that subjects with grade 2/3 diarrhoea developed at any stage during treatment $(41 \%, \mathrm{n}=19)$ have longer OS compared to those without $(\mathrm{P}=0.009)$ [13].

Our results showed that the presentation of diarrhea was correlated with sorafenib treatment efficacy in the SD group (adjusted OR 3.54, 95\% CI $1.70-7.40$ ), but not in the PR group (adjusted OR 2.02, 95\% CI 0.36 - 11.42 ). When cases from the PR and SD groups were pooled for analysis, diarrhea still showed significant correlation with sorafenib treatment efficacy (adjusted OR 3.42, 95\% CI 1.67 - 7.01 ).

Apart from HCC, a similar correlation between the development of AEs and treatment efficacy has been reported in breast cancer patients treated with endocrine therapy as well as in non-small cell lung cancer patients treated with chemotherapy with or without VEGF inhibitors [16-18]. The availability of reliable predictive biomarkers would help identifying which individuals are likely to benefit from antitumoral treatment and to minimize unnecessary toxicity in potentially resistant subjects. In this study on HCC, knowledge on the development of AEs in patients as a surrogate marker of sorafenib efficacy is clinically relevant. Here, we found that the incidences of HFRS and diarrhea are the most predictive markers to sorafenib therapeutic response.

There are some limitations in our study. Firstly, our study is retrospective and patients were presented only at a single tertiary care center. Selection bias likely existed. Secondly, short-term but not long-term prognostic outcomes, such as TTP and OS, were analyzed. Thirdly, patients with any grade of AEs were enrolled, and such self-reported data were subject to some errors. Finally, the status of medical history to viral hepatitis, such as nucleotide/nucleoside analogs (NUCs), interferon or direct-acting antivirals (DAAs), was not recorded nor analyzed. Also, patients were limited to those with cirrhosis Child-Pugh stage A and HCC BCLC stage C. Further prospective research with extended analysis or more variables is needed.

In conclusion, sorafenib-related HFSR and diarrhea are associated with better efficacy as based on short-term radiologic presentations.

\section{References}

1. Llovet JM, Bru C, Bruix J. Prognosis of hepatocellular carcinoma: the BCLC staging classification. Semin Liver Dis. 1999;19(3):329-338.

2. Strumberg D. Preclinical and clinical development of the oral multikinase inhibitor sorafenib in cancer treatment. Drugs Today (Barc). 2005;41(12):773-784.

3. Llovet JM, Ricci S, Mazzaferro V, Hilgard P, Gane E, Blanc JF, de Oliveira AC, et al. Sorafenib in advanced hepatocellular carcinoma. N Engl J Med. 2008;359(4):378-390.

4. Cheng AL, Kang YK, Chen Z, Tsao CJ, Qin S, Kim JS, Luo R, et al. Efficacy and safety of sorafenib in patients in the Asia-Pacific region with advanced hepatocellular carcinoma: a phase III randomised, double-blind, placebocontrolled trial. Lancet Oncol. 2009;10(1):25-34.

5. Llovet JM, Pena CE, Lathia CD, Shan M, Meinhardt G, Bruix J, SHARP Investigators Study Group. Plasma biomarkers as predictors of outcome in patients with advanced hepatocellular carcinoma. Clin Cancer Res. 2012;18(8):2290-2300.

6. Granito A, Marinelli S, Negrini G, Menetti S, Benevento F, Bolondi L. Prognostic significance of adverse events in patients with hepatocellular carcinoma treated with sorafenib. Therap Adv Gastroenterol. 2016;9(2):240-249. 
7. Cho JY, Paik YH, Lim HY, Kim YG, Lim HK, Min YW, Gwak GY, et al. Clinical parameters predictive of outcomes in sorafenib-treated patients with advanced hepatocellular carcinoma. Liver Int. 2013;33(6):950-957.

8. Bettinger D, Schultheiss M, Knuppel E, Thimme R, Blum HE, Spangenberg HC. Diarrhea predicts a positive response to sorafenib in patients with advanced hepatocellular carcinoma. Hepatology. 2012;56(2):789-790.

9. Roberts LR, Sirlin CB, Zaiem F, Almasri J, Prokop LJ, Heimbach JK, Murad MH, et al. Imaging for the diagnosis of hepatocellular carcinoma: A systematic review and meta-analysis. Hepatology. 2018;67(1):401-421.

10. Lencioni R, Llovet JM. Modified RECIST (mRECIST) assessment for hepatocellular carcinoma. Semin Liver Dis. 2010;30(1):52-60.

11. Robert C, Soria JC, Spatz A, Le Cesne A, Malka D, Pautier P, Wechsler J, et al. Cutaneous side-effects of kinase inhibitors and blocking antibodies. Lancet Oncol. 2005;6(7):491-500.

12. Otsuka T, Eguchi Y, Kawazoe S, Yanagita K, Ario K, Kitahara K, Kawasoe H, et al. Skin toxicities and survival in advanced hepatocellular carcinoma patients treated with sorafenib. Hepatol Res. 2012;42(9):879-886.

13. Koschny R, Gotthardt D, Koehler C, Jaeger D, Stremmel W, Ganten TM. Diarrhea is a positive outcome predictor for sorafenib treatment of advanced hepatocellular carcinoma. Oncology. 2013;84(1):6-13.
14. Estfan B, Byrne M, Kim R. Sorafenib in advanced hepatocellular carcinoma: hypertension as a potential surrogate marker for efficacy. Am J Clin Oncol. 2013;36(4):319324.

15. Akutsu N, Sasaki S, Takagi H, Motoya M, Shitani M, Igarashi M, Hirayama D, et al. Development of hypertension within 2 weeks of initiation of sorafenib for advanced hepatocellular carcinoma is a predictor of efficacy. Int J Clin Oncol. 2015;20(1):105-110.

16. Fontein DB, Houtsma D, Hille ET, Seynaeve C, Putter H, Meershoek-Klein Kranenbarg E, Guchelaar HJ, et al. Relationship between specific adverse events and efficacy of exemestane therapy in early postmenopausal breast cancer patients. Ann Oncol. 2012;23(12):3091-3097.

17. Fontein DB, Seynaeve C, Hadji P, Hille ET, van de Water W, Putter H, Kranenbarg EM, et al. Specific adverse events predict survival benefit in patients treated with tamoxifen or aromatase inhibitors: an international tamoxifen exemestane adjuvant multinational trial analysis. J Clin Oncol. 2013;31(18):2257-2264.

18. Goodwin R, Ding K, Seymour L, LeMaitre A, Arnold A, Shepherd FA, Dediu M, et al. Treatment-emergent hypertension and outcomes in patients with advanced non-small-cell lung cancer receiving chemotherapy with or without the vascular endothelial growth factor receptor inhibitor cediranib: NCIC Clinical Trials Group Study BR24. Ann Oncol. 2010;21(11):2220-2226. 